\title{
A fuzzy multiple objective decision support model for energy-economy planning
}

\author{
Ana Rosa Borges ${ }^{\mathrm{a}, \mathrm{b}, *}$, Carlos Henggeler Antunes ${ }^{\mathrm{b}, \mathrm{c}}$ \\ ${ }^{a}$ ISEC-Coimbra Polytechnic Institute, Apartado 10057, Quinta da Nora, 3030-601 Coimbra, Portugal \\ ${ }^{\mathrm{b}}$ INESC-Rua Antero de Quental 199, 3000-033 Coimbra, Portugal \\ ${ }^{\mathrm{c}}$ Department of Electrical Engineering, University of Coimbra, 3030-030 Coimbra, Portugal
}

\begin{abstract}
In this paper an interactive approach to deal with fuzzy multiple objective linear programming problems is presented, which is based on the analysis of the decomposition of the parametric (weight) diagram into indifference regions corresponding to basic efficient solutions. This approach is illustrated to tackle uncertainty and imprecision associated with the coefficients of an input-output energy-economy planning model, aimed at providing decision support to decision makers in the study of the interactions between the energy system and the economy on a national level.
\end{abstract}

(c) 2002 Published by Elsevier Science B.V.

Keywords: Fuzzy multiple objective linear programming; Decision support systems; Interactive methods; Energy system; Input-output analysis

\section{Introduction}

The energy sector is of outstanding importance to the analysis of an economy on a national level, because of direct and indirect consequences on several well-being indicators ranging from economical aspects to social and environmental ones. For some industrialized countries, such as Portugal, energy dependence is a crucial issue because of the high level of imports of primary energy, namely fossil fuels. In these circumstances, wellfounded information concerning economic devel-

\footnotetext{
${ }^{*}$ Corresponding author. Tel.: +351-239-851040; fax: +351239-824692.

E-mail address: arborges@isec.pt (A.R. Borges).
}

opment constrained by limited energy resources must be provided to decision makers (DMs). A decision support model addressing the energy sector in the broader context of the economic system has been developed enabling to study their interactions.

The interactions among different sectors of an economy can be dealt with input-output analysis. In the framework of input-output analysis an economic system is disaggregated into a number of interdependent sectors. Each sector in the static input-output table produces a particular output, with fixed input and output structure, and no substitution between the outputs of the different sectors (Leontieff, 1951). By providing a systemic view of macro-economic aggregates and economic flows in a given economic system, input-output analysis is an useful tool to 
assist in the formulation of economic planning models.

Economy-energy planning problems are characterized by the need of explicitly considering multiple, conflicting, and incommensurate axes of evaluation. Mathematical models for decision support must address, in an explicit manner, aspects of distinct nature such as social, economical, environmental, and technical ones rather than attempting to encompass them in an one-dimensional economic indicator (Zeleny, 1982; Steuer, 1986). Moreover, the multiple objective approach intrinsically possesses a value-added role in the modeling process and in model analysis, supporting reflection and creativity in face of a larger universe of potential solutions rather than a single "optimal" solution.

A multiple objective linear programming (MOLP) model based on input-output analysis has been developed devoted to study the relationships between the economy and the energy sector on a national level. The model allows for the computation of the amount of energy required for the provision of goods and services within an economy, both for intermediate consumption (that is, for sectors producing other goods or services) and directly in final demand. Moreover, by associating the consumption of fossil fuels and the corresponding carbon content with the activity level of each sector it is possible determine the resulting amount of emissions of atmospheric pollutants (such as carbon dioxide).

In a model possessing a great diversity and complexity of input information, which is used to derive the coefficients to the MOLP model, several sources of uncertainty are at stake. These are taken into account herein by considering some model coefficients as triangular fuzzy numbers. Interactive techniques based on the analysis of the decomposition of the parametric (weight) diagram into indifference regions corresponding to basic efficient solutions have been developed and computationally implemented as the core of a decision support system (DSS) to deal with uncertainty in MOLP models.

Section 2 presents some key concepts of MOLP that are important to introduce the proposed visual interactive approach to deal with fuzzy
MOLP problems. This approach is described in detail in Section 3. In Section 4 a multiple objective input-output model for energy planning is briefly presented, which is aimed at studying the energy sector in the context of the economy on a national level. Some illustrative results are reported in Section 5. Finally, in Section 6 some conclusions are drawn.

\section{Multiple objective linear programming}

Let us consider the following MOLP problem with $p$ linear objective functions and $m$ linear constraints:

$$
\begin{array}{ll}
\text { "max" } & \mathbf{z}=C \mathbf{x} \\
\text { s.t. } & \mathbf{x} \in X=\left\{\mathbf{x} \in \mathfrak{R}^{n}: A \mathbf{x}=\mathbf{b}, \mathbf{x} \geqslant \mathbf{0}\right\}
\end{array}
$$

where $A$ is a $m \times n$ matrix, b is the $m$ right-hand side (RHS) column vector and $C$ is a $p \times n$ matrix of objective functions coefficients. "max" denotes the operation of computing efficient solutions.

A feasible solution to (1) is called efficient if and only if no other feasible solution exists that improves one of the objective functions without deteriorating (at least one of the) other objective functions. A relaxed notion is also generally used: a feasible solution is called weakly efficient if and only if there is no other feasible solution that strictly improves all objective function values. For definitions and mathematical details see, for instance, Steuer (1986).

Let $C_{r .}(r=1, \ldots, p)$ be the $r$ th row of $C$. When solving problem (1) by the weighted-sum approach, each objective $C_{r .} \mathbf{x}$ is associated with a positive weight $\lambda_{r}$ (an $\lambda_{r}=0$ could lead to a weakly efficient solution). Without loss of generality, each weighting vector can be normalized so that its elements sum to one:

$$
\begin{aligned}
\Lambda=\left\{\boldsymbol{\lambda}: \boldsymbol{\lambda}=\left(\lambda_{1}, \lambda_{2}, \ldots \lambda_{p}\right) \in \mathfrak{R}^{p},\right. \\
\left.\sum_{r=1}^{p} \lambda_{r}=1, \lambda_{r}>0, r=1, \ldots, p\right\} .
\end{aligned}
$$

Therefore, basic efficient solutions can be obtained by optimizing a scalarizing function consisting of a weighted sum of the objective functions: 
$\max \sum_{r=1}^{p} \lambda_{r}\left(C_{r} \mathbf{x}\right)$

s.t. $\quad \mathbf{x} \in X, \quad \lambda \in \Lambda$.

Let $K$ be the index set of the $(n-m)$ non-basic variables associated with an optimal solution to (3), which is an efficient basic solution to (1), which has been computed by using a given weighting vector. $B$ and $N$ are the submatrices of $A$ corresponding to the basic and non-basic variables, respectively, and $C_{B}\left(\mathbf{x}_{B}\right)$ and $C_{N}\left(\mathbf{x}_{N}\right)$ are the submatrices (subvectors) of $C(\mathbf{x})$ corresponding to the basic and non-basic variables, respectively. An indifference region for the weights (set of weighting vectors that leads to the same basic efficient solution) is defined in $\Lambda$ and can be achieved by the intersection of the $n-m$ hyper-halfspaces resulting from the reduced cost matrix of a multiobjective simplex tableau (Steuer, 1986) associated with a basic efficient solution $\left(W=C_{B} B^{-1} N-C_{N}\right)$, that is

$\bigcap_{k \in K}\left\{\sum_{r=1}^{p} \lambda_{r} w_{r k} \geqslant 0\right\}, \quad \lambda \in \Lambda$.

$w_{r k}$ is the $(r, k)$ element of the reduced cost matrix with respect to objective function $r=1, \ldots, p$, and the non-basic variable $k \in K$. The DM may be indifferent to all combinations of weighting vectors within it because they lead to the same basic effi- cient solution. These indifference regions are defined in a geometrical $(p-1)$-dimensional simplex in a $p$-dimensional Euclidean space.

For three objective functions the use of visual interactive graphical tools are particularly suited for the exchange of information with the DM. The decomposition of $\Lambda$ into indifference regions lends itself well to a progressive and selective learning of the efficient solution set in MOLP (Clímaco and Antunes, 1987, 1989).

Fig. 1 shows a three-dimensional weight space where the hatched polygon is the indifference region associated with the basic efficient solution computed by optimizing the weighted-sum LP considering the weighting vector $\lambda=P$. Each of the $n-m(n-m=4$ in this example) halfspaces defined in (4) corresponds to a non-basic variable. $\Pi_{k}$ is the plane obtained from the $k$ halfspace in (4) replacing the inequality ' $\geqslant$ ' by ' $=$ ' and it is defined by $\sum_{r=1}^{3} \lambda_{r} w_{r k}=0 . \pi_{k}$ denotes the intersection of $\Pi_{k}$ with $\Lambda$.

In the operational framework of the proposed interactive fuzzy MOLP approach, the parametric diagram, a geometrical two-dimensional simplex in a three-dimensional Euclidean space, is used to display relevant information in the same graph to the DM. This enables the DM to visualize dynamically and interactively the behavior of efficient solutions according to changes in the initial model coefficients and DM's preferences.

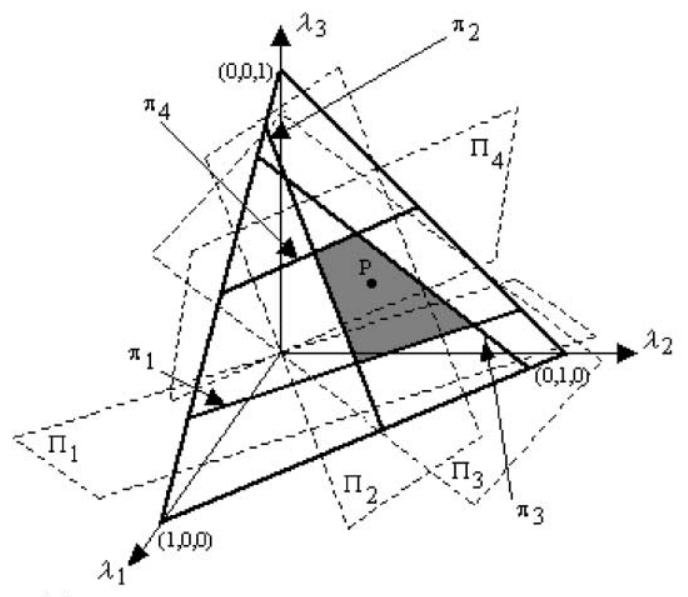

(a)

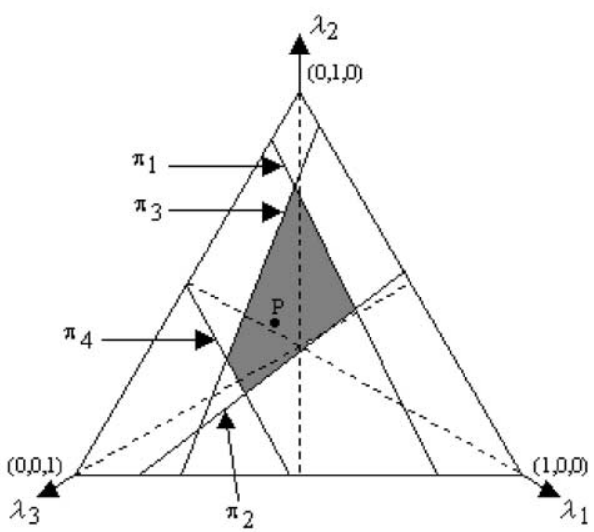

(b)

Fig. 1. Decomposition of $\Lambda$ into indifference regions. 


\section{Fuzzy analysis in MOLP}

A great diversity of possible modifications to the classical (crisp) LP problem (1) have been proposed in a fuzzy environment and different ways to deal with the corresponding types of fuzziness in LP models are reported in the literature.

The coefficients of the vector $\mathbf{b}$ or the matrices $C$ or $A$ can have a fuzzy character (Tanaka and Asai, 1984; Carlsson and Korhonen, 1986; Sakawa and Yano, 1990) either because they are fuzzy in nature or their perception is fuzzy.

The mathematical relations involved may also be fuzzy (fuzzy objectives and/or constraints) (Zimmermann, 1978, 1983; Chanas, 1983; Werners, 1987a,b). The DM may not be interested in optimizing some of the objective functions; rather he/she might want to "improve" as much as possible their values in order to reach some "aspiration levels" which may not be crisply defined. The constraints may also be fuzzy, that is the ' $=$ ' sign might not be met in the strictly mathematical sense but the DM may accept small violations on it.

Moreover, the solution of a fuzzy linear programming problem may be crisp (Zimmermann, 1978, 1983; Tanaka and Asai, 1984; Werners, 1987a,b; Sakawa and Yano, 1990) or fuzzy (Chanas, 1983; Carlsson and Korhonen, 1986). In the latter case a solution set (of all fuzzy efficient solutions) is presented to the DM and he/she must choose the one that is more in accordance with his/ her preferences.

In this study the objective function coefficients and the constraints' RHS as well as the coefficients in the technological matrix and in the objective functions associated with a new decision variable are considered fuzzy coefficients and are charac-

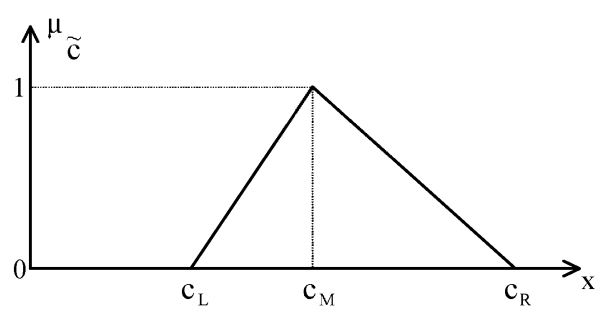

Fig. 2. A triangular membership function. terized by triangular membership functions defined by (Fig. 2):

$$
\mu_{\tilde{c}}(x)= \begin{cases}0 & \text { if } x \leqslant c_{\mathrm{L}}, \\ \frac{\left(x-c_{\mathrm{L}}\right)}{\left(c_{\mathrm{M}}-c_{\mathrm{L}}\right)} & \text { if } x \in] c_{\mathrm{L}}, c_{\mathrm{M}}[, \\ 1 & \text { if } x=c_{\mathrm{M}}, \\ \frac{\left(c_{\mathrm{R}}-x\right)}{\left(c_{\mathrm{R}}-c_{\mathrm{M}}\right)} & \text { if } x \in] c_{\mathrm{M}}, c_{\mathrm{R}}[, \\ 0 & \text { if } x \geqslant c_{\mathrm{R}} .\end{cases}
$$

A triangular fuzzy number can be denoted as $\tilde{c}=\left(c_{\mathrm{L}}, c_{\mathrm{M}}, c_{\mathrm{R}}\right)$ where $c_{\mathrm{M}}$ is the central value (maximum grade of membership), $c_{\mathrm{M}}-c_{\mathrm{L}}$ is the left spread and $c_{R}-c_{M}$ is the right spread.

Interactive techniques to deal with fuzzy MOLP models have been developed and implemented as the core of a DSS. The DM can visualize dynamically the changes in the indifference regions corresponding to the initial (crisp) basic efficient solutions and compute new basic efficient solution(s) in an interactive manner by varying continuously the grades of membership as well as by changing the values of $c_{\mathrm{L}}$ and $c_{\mathrm{R}}$ (and also the value of $c_{M}$ when introducing a new variable) for each fuzzy coefficient. The new basic efficient solutions are computed by using the Simplex or Dual-Simplex method starting from the multiobjective Simplex tableaux corresponding to an efficient solution previously computed with maximum grade of membership and considering weighting vectors within regions of $\Lambda$ not yet filled with indifference regions (Borges and Antunes, 2000).

The aim of the proposed interactive DSS is to help the DM to exploit the uncertainty associated with the initial problem, modeled by means of fuzzy numbers, to gather further knowledge on the problem as well as to reinforce or weaken his/her own convictions and preferences in order to make a better informed decision. During the interactive study the DM is always allowed to revise prior preference information and exploit new search directions.

\subsection{Objective function matrix}

The objective function coefficients are defined as triangular fuzzy numbers where the central 
values $\left(c_{\mathrm{M}}\right)$ are the crisp model parameters, and the grade of membership $(y)$ is the same for all objective functions.

For each different $y$ only the objective function coefficients are changing. Therefore, the extreme points of the feasible region remain unchanged. However, the reduced cost matrix values, $W=$ $C_{B} B^{-1} N-C_{N}$, vary and the efficient region can eventually change. The indifference regions corresponding to the basic efficient solutions to the initial problem are then changing continuously, in size and shape, with changes of the grade of membership.

New basic efficient solutions can be computed by using weighing vectors within regions of $\Lambda$ not yet filled with indifference regions. The new basic efficient solutions are computed by using the Simplex method starting from the multiobjective Simplex tableaux corresponding to an efficient solution previously computed with maximum grade of membership (corresponding to the $c_{\mathrm{M}}$ value) and selected by the DM.

The indifference regions associated with the computed basic efficient solutions can even disappear meaning that the corresponding extreme point becomes dominated.

\subsection{Right-hand side}

The constraints' RHS coefficients are defined as triangular fuzzy numbers, with the central value $\left(c_{\mathrm{M}}\right)$ corresponding to the crisp model parameters, and the grade of membership $(t)$ is the same for all constraints.

The values of the $\mathbf{b}$ vector are changing with $t$ and so the feasible region changes. If some decision variable values regarding a basic efficient solution, $\mathbf{x}_{B}=B^{-1} \mathbf{b}$, become negative, then the corresponding solution becomes infeasible. Since the objective function coefficients remain unchanged, then for a given basis the reduced cost matrix $W=C_{B} B^{-1} N-C_{N}$ does not change and the optimality condition is never violated. Therefore, the indifference regions corresponding to the basic efficient solutions do not change continuously, as in the case of objective functions, but they change 'suddenly' as they appear or disappear, meaning that the corresponding efficient basis becomes feasible or infeasible, respectively.
New basic efficient solutions can be computed by using weighing vectors within regions of $\Lambda$ not yet filled with indifference regions by using the Dual-Simplex method and starting from the multiobjective Simplex tableaux corresponding to an initial basic efficient solution. The starting efficient solution is such that the selected weighing vector belongs to the initial corresponding indifference region.

If the selected weighing vector is within regions of $\Lambda$ not initially filled with indifference regions then the DM is asked to previously compute the corresponding initial basic efficient solution (computed with maximum grade of membership value, that is considering the $c_{\mathrm{M}}$ values).

\subsection{Introduction of new decision variables}

The coefficient vectors in the objective functions and in the technological matrix of a new decision variable are defined as triangular fuzzy numbers, $\tilde{C}_{x_{\text {new }}}$ and $\tilde{A}_{x_{\text {new }}}$, respectively. Two distinct grades of membership are considered: one for all the objective functions and another one for all constraints.

When both grades of membership are changing the reduced cost matrix column regarding the new variable $\left(W_{x_{\text {new }}}=C_{B} B^{-1} \tilde{A}_{x_{\text {new }}}-\tilde{C}_{x_{\text {new }}}\right)$, must satisfy

$\left\{\lambda^{\mathrm{T}}\left(C_{B} B^{-1} \tilde{A}_{x_{\text {new }}}-\tilde{C}_{x_{\text {new }}}\right) \geqslant 0\right\}, \quad \lambda \in \Lambda$,

for the basic solution under analysis to remain efficient.

The introduction of a new decision variable into a MOLP leads to the creation of new extreme points with non-zero value in the new variable as well as new edges and faces (Antunes and Clímaco, 1992). The new variable may be classified, with respect to a selected basic efficient solution, as:

- non-efficient variable, whenever (5) does not affect the initial (4) associated with the selected efficient solution ((5) is redundant with respect to the initial (4));

- efficient variable, whenever (5) does intersect the initial (4) associated with the selected efficient solution;

- "must be made basic" variable, because the selected efficient solution becomes dominated (the initial (4) associated with the selected effi- 
cient solution does not belong to the hyper-halfspace defined by (5)).

A non-basic variable is efficient with respect to a given efficient basis if and only if when introduced into the basis it leads to an adjacent efficient basis through an efficient edge. In this situation, in addition to "update" the selected basic efficient solution (that is, to compute the intersection of the initial (4) with (5) to determine the new indifference region), new basic efficient solutions with non-zero value in the new variable can be computed.

If the variable "must be made basic" new basic efficient solutions with non-zero value in the new variable can be computed.

The new basic efficient solutions are computed as described in Section 3.1.

Whenever the introduction of new decision variables is considered, the triangular membership functions associated with the parameters (corresponding to the new variable) can be changed by modifying not only the corresponding $c_{\mathrm{L}}$ and $c_{\mathrm{R}}$ but also the $c_{M}$ values.

\section{An input-output MOLP model for energy- economy planning}

An input-output table based on statistical data available from several Portuguese and international sources has been developed which considers 21 economic sectors. The energy sector components have been disaggregated in detail, allowing the distinction between primary and secondary energy sources, by means of 23 artificial sectors that are used for distributing the output of the oil refining sector and the by-products through the consuming sectors. Energy flows (in toes, tons of oil equivalent) and monetary flows (in monetary units) are considered. The anatomy of the inputoutput model is as follows: a $(44 \times 44)$ matrix with the inter- and intra-sector flows, six column vectors with the components of final demand (private consumption, collective consumption, gross fixed capital formation, positive and negative stock changes, and exports), one column vector for the competitive imports and three row vectors for the primary inputs (wages, net indirect taxes, and operating surplus).

The consumption of fossil fuels is associated with the level of activity of each sector, enabling to evaluate the embodied energy required to manufacture a good or service. The analysis is then extended to account for emissions of air pollutants resulting from the burning of fossil fuels by incorporating the requirements of primary energy for the economic activities. Total emissions from each sector and the whole economy can be computed by using coefficients that relate the amount of carbon dioxide produced per unit of fuel consumed (through its calorific value). The top-down methodology proposed by the Intergovernmental Panel for Climate Change (IPCC, 1996) has been used to model carbon dioxide $\left(\mathrm{CO}_{2}\right)$ emissions, which is based on the principles of combustion and composition of fuels.

The model considers three objective functions:

- energy imports (to be minimized, taking into account the energy dependence of the country);

- self-production of electricity (to be maximized, in order to encourage the use of alternative forms of energy, valuing the recycle of wastes and allowing both energy economies and the minimization of waste disposal);

- $\mathrm{CO}_{2}$ emissions (to be minimized, due to the impact of energy resources on the environment, specifically air pollution).

Energy imports and self-production of electricity are expressed in physical units of energy (toes) and $\mathrm{CO}_{2}$ emissions is in $\mathrm{Gg}$.

Several categories of constraints are considered in the MOLP model:

- balance of payments (to guarantee a certain level of external equilibrium);

- public deficit (to take into account European Union requirements);

- upper and lower bounds on the production capacity of each activity sector;

- upper and lower bounds on imports and exports (to avoid an over-specialization since they are not linked to the model coefficients); 
- storage capacity and security stocks for hydrocarbons (to guarantee that positive stock changes never exceed storage capacity and negative stock changes are never below security stocks);

- coherence constraints for goods and services (imposing that the use of a specific good or service, for intermediate consumption and final demand, cannot exceed the resources available, resulting from national production and competitive imports);

- defining constraints for gross added value (an indicator enabling to quantify the resources generated within the country) and gross domestic product (both according to expense and product definitions).

Please see Oliveira and Antunes (2000) and Antunes et al. (2002) for further details on the input-output structure and the mathematical model.

\section{Some illustrative results}

After performing a progressive and selective learning of the efficient solution set in a crisp environment, the DM is given the possibility of interactively studying the effects of the fuzziness arising in the parameters of the objective functions, the constraints' RHS or in the objective functions and constraints of a new decision variable. Different membership functions (associated with the fuzzy parameters) can be considered by the DM and for each set of them the grades of membership can be continuously changed. The comparative graphical analysis of the decomposition of the parametric (weight) diagram into indifference regions corresponding to the initial (crisp) basic efficient solutions and the new ones computed in a fuzzy environment, as well as the numerical values provided by the DSS, enable the DM to study the fuzzy efficient solution set.

Firstly, a search for basic efficient solutions has been progressively performed in order to have an overview of solutions with different characteristics for the energy-economy planning model. Surprisingly, $\Lambda$ become completely filled with few indif- ference regions, as displayed in Fig. 3, meaning that, in crisp environment, all basic efficient solutions have been found. The objective function values (as well as the indifference region areas) of those solutions are shown in Table 1.

In general, the aim is to compute "well-dispersed" solutions to gain some insights into the problem, which a further selective search could be based on. This usually happens for medium-sized MOLP problems in which hundreds of basic efficient solutions can be found. In particular, it is the case of models similar to the one herein presented but considering other objective functions (Oliveira and Antunes, 2000; Antunes et al., 2002).

Fig. 3 and the further ones are actual copies of the screens presented to the user.

Let us take into account the effect of the uncertainty associated with the objective function coefficients modeled as triangular fuzzy numbers on the behavior of efficient solutions. Let us suppose that the DM considers solution 5 (previously computed with maximum grade of membership) as a good compromise solution and he/she is interested in studying its stability regarding changes of the grade of membership.

The DM can dynamically visualize the changes (in size and shape) of the indifference regions corresponding to the initial computed basic efficient solutions by changing the grade of member-

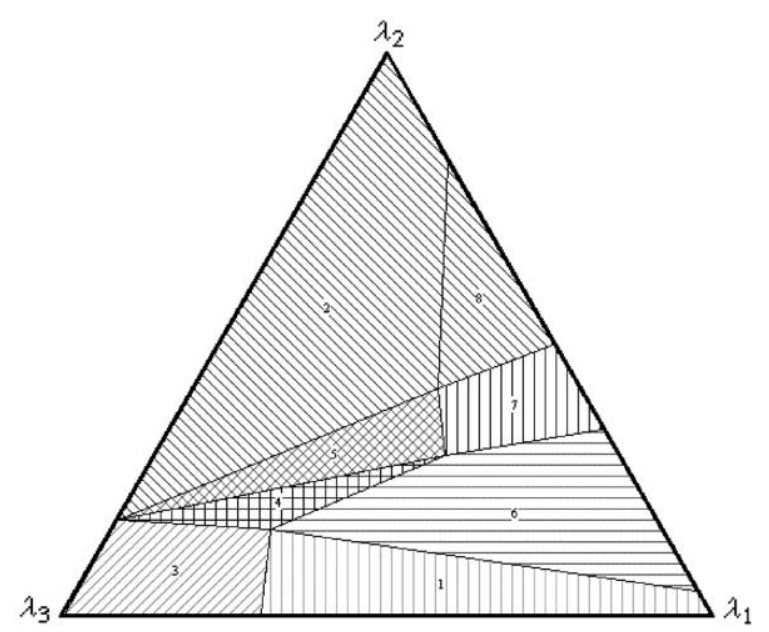

Fig. 3. Indifference regions corresponding to the initial basic efficient solutions. 
Table 1

Initial basic efficient solutions

\begin{tabular}{llrrr}
\hline Solution & Energy imports & Self-production of electricity & $\mathrm{CO}_{2}$ emissions & Area (\%) \\
\hline 1 & 12271100 & 179513 & 1591600 & 12.8149 \\
2 & 87769700 & 22622700 & 30606300 & 35.5831 \\
3 & 12727600 & 164322 & 1246240 & 8.7248 \\
4 & 13222500 & 1334770 & 2776380 & 3.6413 \\
5 & 14844100 & 2187230 & 3890810 & 6.2979 \\
6 & 12395100 & 986354 & 2799170 & 19.4846 \\
7 & 14592700 & 2182660 & 4589630 & 6.3779 \\
8 & 85810100 & 22499000 & 34996400 & 7.0706 \\
\hline
\end{tabular}

ship $y$ (objective functions). New solutions corresponding to regions of $\Lambda$ not yet filled that the DM is interested in studying can also be computed.

For instance, with $y=0.0500_{\mathrm{R}}$ efficient solutions $1,6,7$ and 8 previously computed become dominated (their corresponding indifference regions disappear) and it is possible to calculate 6 new basic efficient solutions $(\mathrm{L}-\mathrm{Q})$ as displayed in Fig. 4(a) (Table 2). Notice that the areas of the indifference regions corresponding to the efficient solutions computed in crisp environment have changed.

If the DM is not interested in some solutions they can be disregarded further on. Once more the DM may conclude, namely by analyzing the objective function values of the relevant solutions, that certain regions of $\Lambda$ are not worthwhile to search.
For example, in Fig. 4(b) solutions L, O and Q are eliminated because the DM may consider that the last one has a high value for energy imports and the other ones have small values with respect to self-production of electricity.

If the grade of membership $y$ is changed from $0.0_{\mathrm{L}}$ to $0.0_{\mathrm{R}}$ the results presented in Table 3 are obtained. Even though the core idea behind our approach is not to perform an exhaustive study of all basic efficient solutions it has been done for the sake of illustration and because it is not computationally heavy.

In Fig. 5(a) and (b) the decompositions of the weight diagram for $y=0.0000_{\mathrm{L}}$ and $y=0.0000_{\mathrm{R}}$ are displayed.

With $y=0.0000_{\mathrm{L}}$ the efficient solution 7 previously computed becomes dominated and the new basic efficient solutions A-I can be reached.

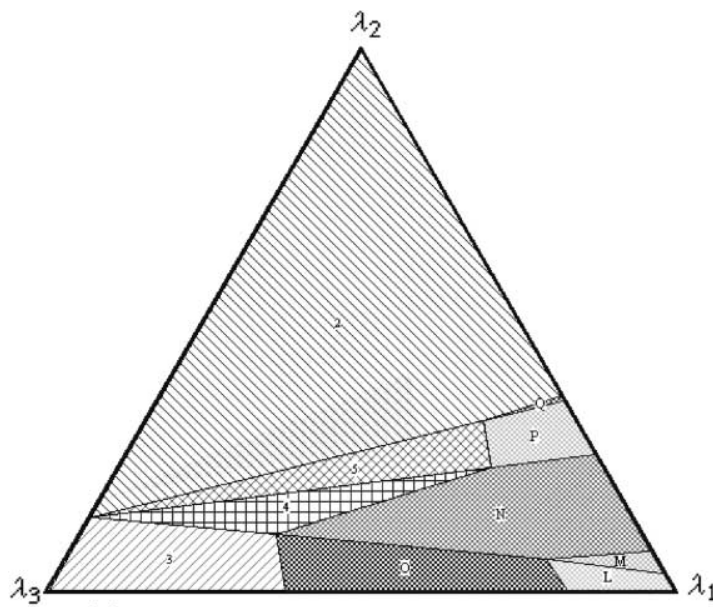

(a)

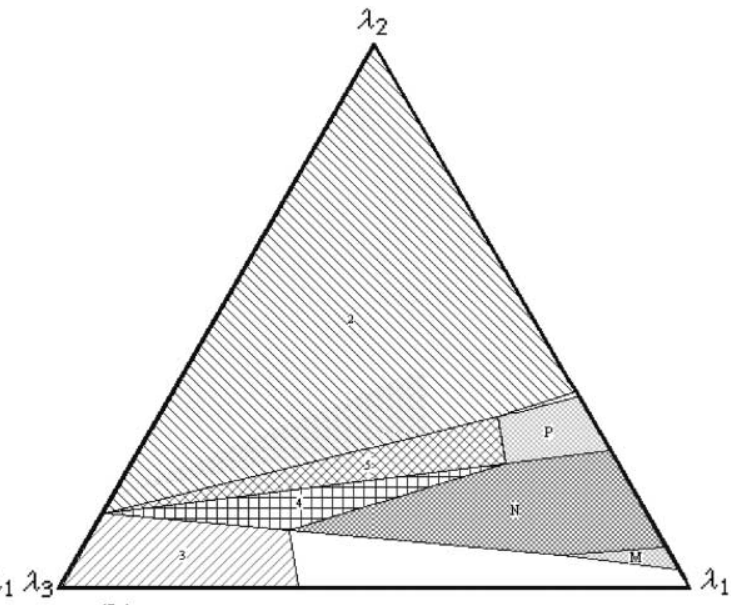

(b)

Fig. 4. Fuzzy analysis of the objective function coefficients $\left(y=0.0500_{\mathrm{R}}\right)$. 
Table 2

Fuzzy analysis of the objective function coefficients $\left(y=0.0500_{\mathrm{R}}\right)$

\begin{tabular}{|c|c|c|c|c|c|c|}
\hline \multicolumn{2}{|c|}{ Solution $\left(y=0.0500_{\mathrm{R}}\right)$} & \multirow{2}{*}{$\begin{array}{l}\text { Energy imports } \\
87769700\end{array}$} & \multirow{2}{*}{$\begin{array}{l}\text { Self-production } \\
\text { of electricity }\end{array}$} & \multirow{2}{*}{$\begin{array}{l}\mathrm{CO}_{2} \text { emissions } \\
30606300\end{array}$} & \multirow{2}{*}{$\begin{array}{l}\text { Area }(\%) \\
35.5831\end{array}$} & \multirow{2}{*}{$\begin{array}{l}\text { Number of } \\
\text { iterations }\end{array}$} \\
\hline 2 & Crisp & & & & & \\
\hline & Fuzzy & 59014100 & 25846400 & 27751500 & 56.0235 & - \\
\hline \multirow[t]{2}{*}{3} & Crisp & 12727600 & 164322 & 1246240 & 8.7248 & - \\
\hline & Fuzzy & 10477700 & 187738 & 1148910 & 8.1414 & - \\
\hline \multirow[t]{2}{*}{4} & Crisp & 13222500 & 1334770 & 2776380 & 3.6413 & - \\
\hline & Fuzzy & 10668100 & 1524980 & 2496820 & 4.8045 & - \\
\hline \multirow[t]{2}{*}{5} & Crisp & 14844100 & 2187230 & 3890810 & 6.2979 & - \\
\hline & Fuzzy & 11888100 & 2498910 & 3478520 & 5.6871 & - \\
\hline \multirow[t]{2}{*}{$\mathrm{L}$} & Crisp & 12272100 & 179509 & 1592770 & Non-efficient & \\
\hline & Fuzzy & 10438400 & 205203 & 1476560 & 1.4773 & 11 \\
\hline \multirow[t]{2}{*}{ M } & Crisp & 12396200 & 985552 & 2799340 & Non-efficient & \\
\hline & Fuzzy & 10550100 & 1125990 & 2549910 & 0.7168 & 8 \\
\hline \multirow[t]{2}{*}{$\mathrm{N}$} & Crisp & 13223400 & 1333860 & 2776560 & Non-efficient & \\
\hline & Fuzzy & 10666600 & 1523940 & 2497050 & 13.0926 & 8 \\
\hline \multirow[t]{2}{*}{$\mathrm{O}$} & Crisp & 12728400 & 164196 & 1247130 & Non-efficient & \\
\hline & Fuzzy & 10476900 & 187822 & 1149740 & 6.9128 & 7 \\
\hline \multirow[t]{2}{*}{$\mathrm{P}$} & Crisp & 14846700 & 2187210 & 3892150 & Non-efficient & \\
\hline & Fuzzy & 11887900 & 2498890 & 3479770 & 2.9264 & 2 \\
\hline \multirow[t]{2}{*}{ Q } & Crisp & 87785700 & 22620800 & 30805200 & Non-efficient & \\
\hline & Fuzzy & 59009500 & 25844300 & 27750600 & 0.1531 & 2 \\
\hline
\end{tabular}

Table 3

Fuzzy analysis of the objective function coefficients

\begin{tabular}{llll}
\hline & $y \in$ & Initial efficient solutions & New efficient solutions \\
\hline (i) & {$\left[0.0000_{\mathrm{L}} ; 0.030_{\mathrm{L}}[\right.$} & $1,2,3,4,5,6,8$ & $\mathrm{~A}, \mathrm{~B}, \mathrm{C}, \mathrm{D}, \mathrm{E}, \mathrm{F}, \mathrm{G}, \mathrm{H}, \mathrm{I}$ \\
(ii) & {$\left[0.0300_{\mathrm{L}} ; 0.0367_{\mathrm{L}}[\right.$} & $1,2,3,4,5,6,7,8$ & $\mathrm{~A}, \mathrm{~B}, \mathrm{D}, \mathrm{E}, \mathrm{F}, \mathrm{G}, \mathrm{H}, \mathrm{I}$ \\
(iii) & {$\left[0.0367_{\mathrm{L}} ; 0.1733_{\mathrm{L}}[\right.$} & $1,2,3,4,5,6,7,8$ & $\mathrm{~A}, \mathrm{~B}, \mathrm{D}, \mathrm{E}, \mathrm{F}, \mathrm{G}, \mathrm{I}, \mathrm{J}$ \\
(iv) & {$\left[0.1733_{\mathrm{L}} ; 0.1767_{\mathrm{L}}[\right.$} & $1,2,3,4,5,6,7,8$ & $\mathrm{~A}, \mathrm{~B}, \mathrm{D}, \mathrm{E}, \mathrm{F}, \mathrm{G}, \mathrm{J}$ \\
(v) & {$\left[0.1767_{\mathrm{L}} ; 0.1867_{\mathrm{L}}[\right.$} & $1,2,3,4,5,6,7,8$ & $\mathrm{~A}, \mathrm{~B}, \mathrm{D}, \mathrm{E}, \mathrm{J}$ \\
(vi) & {$\left[0.1867_{\mathrm{L}} ; 0.5133_{\mathrm{L}}[\right.$} & $1,2,3,4,5,6,7,8$ & $\mathrm{~A}, \mathrm{~B}, \mathrm{D}, \mathrm{J}$ \\
(vii) & {$\left[0.5133_{\mathrm{L}} ; 0.5867_{\mathrm{L}}[\right.$} & $1,2,3,4,5,6,7,8$ & $\mathrm{~A}, \mathrm{~B}, \mathrm{~J}$ \\
(viii) & {$\left[0.5867_{\mathrm{L}} ; 0.6100_{\mathrm{L}}[\right.$} & $1,2,3,4,5,6,7,8$ & $\mathrm{~A}, \mathrm{~J}$ \\
(ix) & {$\left[0.6100_{\mathrm{L}} ; 0.6500_{\mathrm{L}}[\right.$} & $1,2,3,4,5,6,7,8$ & $\mathrm{~A}$ \\
(x) & {$\left[0.6500_{\mathrm{L}} ; 0.7200_{\mathrm{R}}[\right.$} & $1,2,3,4,5,6,7,8$ & \\
(xi) & {$\left[0.7200_{\mathrm{R}} ; 0.7133_{\mathrm{R}}[\right.$} & $1,2,3,4,5,6,7$ & $\mathrm{~L}, \mathrm{M}$ \\
(xii) & {$\left[0.7133_{\mathrm{R}} ; 0.5400_{\mathrm{R}}[\right.$} & $1,2,3,4,5,6$ & $\mathrm{~L}, \mathrm{M}, \mathrm{N}$ \\
(xiii) & {$\left[0.5400_{\mathrm{R}} ; 0.3167_{\mathrm{R}}[\right.$} & $1,2,3,4,5,6$ & $\mathrm{~L}, \mathrm{M}, \mathrm{N}$ \\
(xiv) & {$\left[0.3167_{\mathrm{R}} ; 0.3100_{\mathrm{R}}[\right.$} & $1,2,3,4,5,6$ & $\mathrm{~L}, \mathrm{M}, \mathrm{N}, \mathrm{O}$ \\
(xv) & {$\left[0.3100_{\mathrm{R}} ; 0.3067_{\mathrm{R}}[\right.$} & $1,2,3,4,5$ & $\mathrm{~L}, \mathrm{M}, \mathrm{N}, \mathrm{O}$ \\
(xvi) & {$\left[0.3067_{\mathrm{R}} ; 0.2967_{\mathrm{R}}[\right.$} & $1,2,3,4,5$ & $\mathrm{~L}, \mathrm{M}, \mathrm{N}, \mathrm{O}, \mathrm{P}$ \\
(xvii) & {$\left[0.2967_{\mathrm{R}} ; 0.1200_{\mathrm{R}}[\right.$} & $2,3,4,5$ & $\mathrm{~L}, \mathrm{M}, \mathrm{N}, \mathrm{O}, \mathrm{P}, \mathrm{Q}$ \\
(xviii) & {$\left[0.1200_{\mathrm{R}} ; 0.1133_{\mathrm{R}}[\right.$} & $2,3,4,5$ & \\
(xix) & {$\left[0.1133_{\mathrm{R}} ; 0.0000_{\mathrm{R}}\right]$} & $2,3,4,5$ & \\
\hline
\end{tabular}




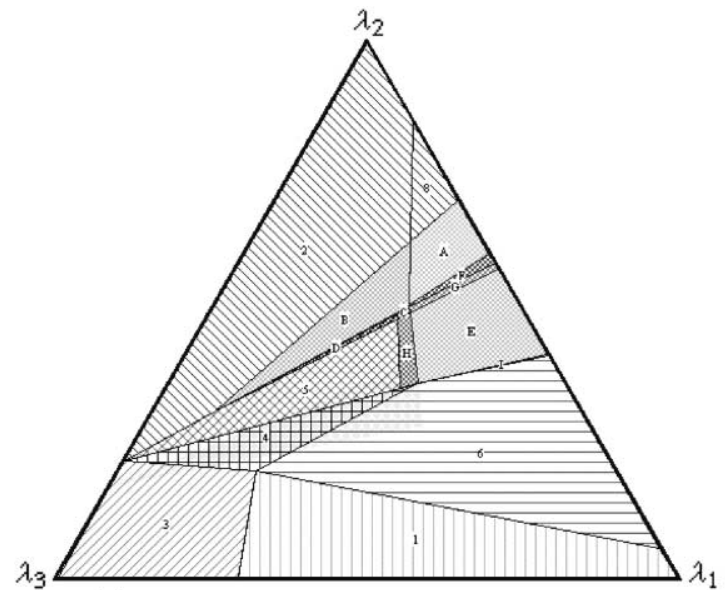

(a) $\left(y=0.0000_{\mathrm{L}}\right)$

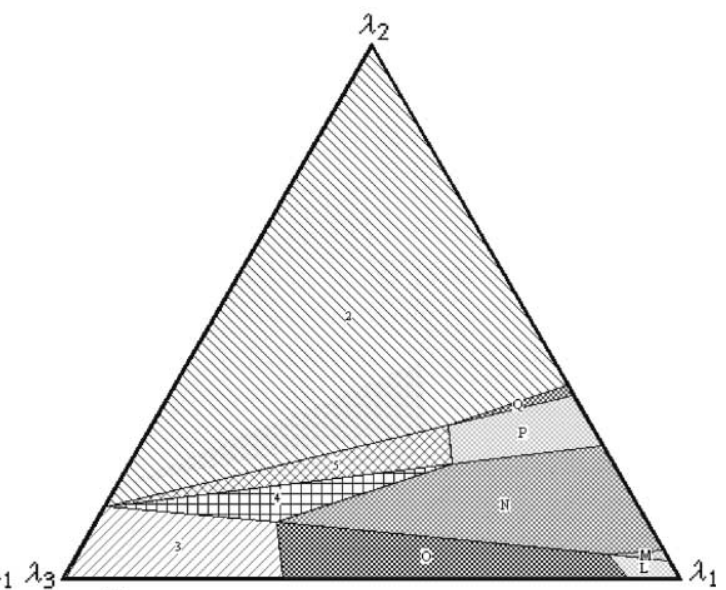

(b) $\left(y=0.0000_{\mathrm{R}}\right)$

Fig. 5. Fuzzy analysis of the objective function coefficients. (a) $\left(y=0.0000_{\mathrm{L}}\right)$, (b) $\left(y=0.0000_{\mathrm{R}}\right)$.

For $y=0.0000_{\mathrm{R}}$ the efficient solutions $1,6,7$ and 8 previously computed become dominated and the new basic efficient solutions L-P can be reached. The solutions that can be reached with $y=0.0000_{\mathrm{R}}$ and $y=0.0500_{\mathrm{R}}$ are the same. Nevertheless, the areas of the indifference regions corresponding to the efficient solutions $3,4,5, \mathrm{~L}$ and $\mathrm{M}$ become smaller than the ones in Fig. 4(a); the areas corresponding to the efficient solutions 2 , $\mathrm{N}, \mathrm{O}, \mathrm{P}$ and $\mathrm{Q}$ become greater than the ones in Fig. 4(a).

Having in mind the definition of $\alpha$-level set (Zadeh, 1965; Zimmermann, 1987, 1992) it can also be envisaged a different way of analyzing the results presented in Table 3. Often the DM may be interested in knowing the computed basic efficient solutions which are obtained if the grade of membership $y$ is at least $\alpha$. For instance, considering a grade of membership above 0.5133 the solutions A, B, J, L, M and $1-8$ can be reached (Fig. 6(a-f), and ranges (vii)-(xiii) in Table 3). For a grade of membership above 0.65 only the crisp basic efficient solutions 1 to 8 can be obtained (ranges (x)-(xii) in Table 3).

If the DM wants to proceed the fuzzy analysis of the objective function coefficients he/she could select another crisp basic efficient solutions (initially computed with maximum grade of membership) as the "starting" one and/or change some of the triangular membership functions associated with those parameters (by modifying the corresponding $c_{\mathrm{L}}$ and $c_{\mathrm{R}}$ values) and perform a similar study.

Let us suppose that the DM also wants to study the behavior of initial basic efficient solutions if some of the RHS coefficients are triangular fuzzy numbers. He/she can dynamically visualize the changes in size of the indifference regions, by changing the grade of membership $t$.

For instance, with $t=0.3367_{\mathrm{R}}$ (Fig. 7 and Table 4) the efficient solutions 1, 3, 5, 6 and 7 become infeasible and it is possible to calculate 8 new basic efficient solutions (A, D, E, F, G, H, I and J).

The grade of membership $t$ can be changed from $0.0_{\mathrm{L}}$ to $0.0_{\mathrm{R}}$ and the results presented in Table 5 are obtained. Considering those membership functions no changes happen for $t \in$ $\left[0.0_{\mathrm{L}} ; 1.0[\right.$.

As for the case of the objective function coefficients, the DM may also be interested in knowing which of the computed efficient bases are obtained if the grade of membership $t$ is at least $\alpha$. For instance, considering a grade of membership above 0.6 the bases associated with solutions E, F, J, L and 1 to 8 can be reached (ranges (i) to (iii) on Table 5). For a grade of membership above 0.9333 only the bases associated with the crisp efficient solutions 1 to 8 are obtained (range (i) on Table 5).

Similarly, if the DM wants to proceed the fuzzy analysis of the RHS coefficients he/she could 


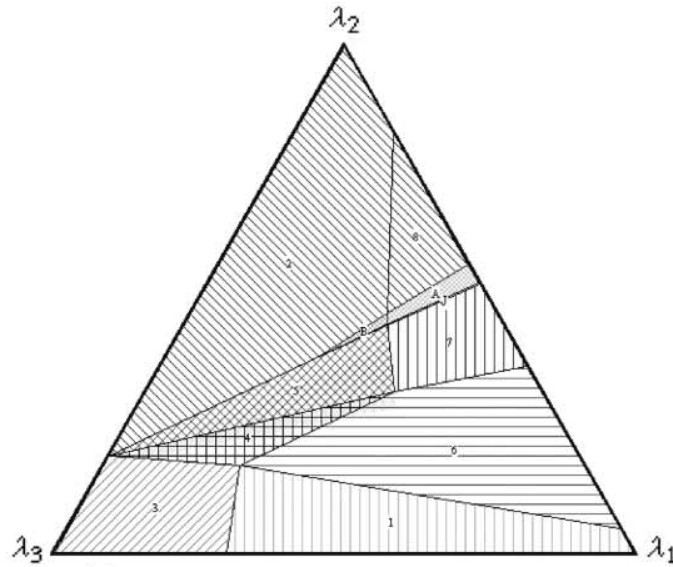

(a) $\left(y=0.5133_{\mathrm{L}}\right)$

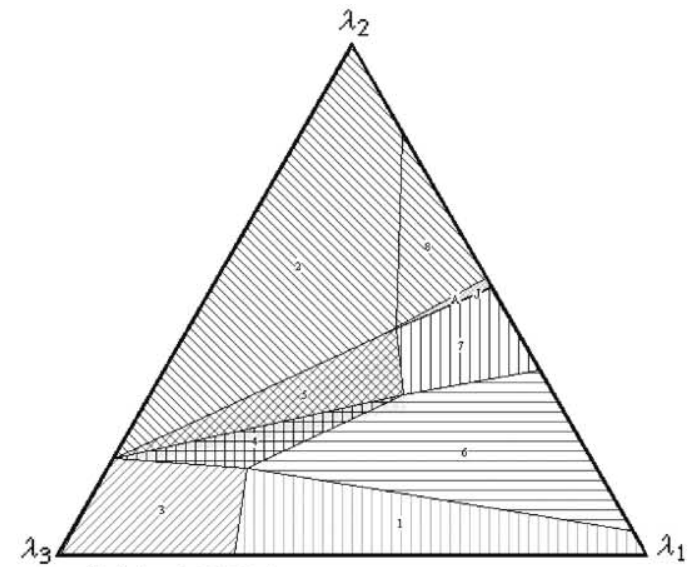

(b) $\left(y=0.5867_{\mathrm{L}}\right)$

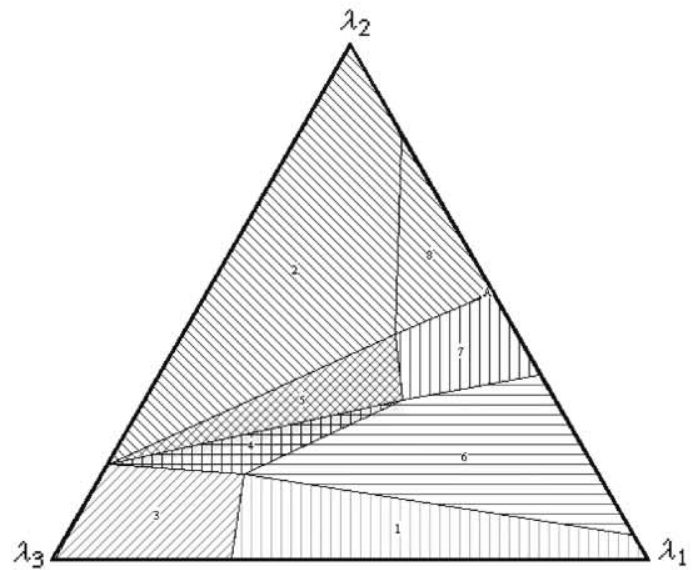

(c) $\left(y=0.6467_{\mathrm{L}}\right)$

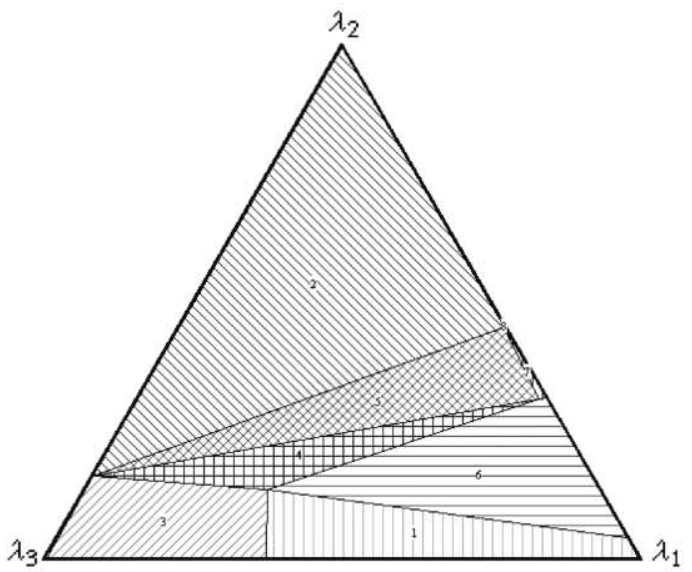

(d) $\left(y=0.7233_{\mathrm{R}}\right)$

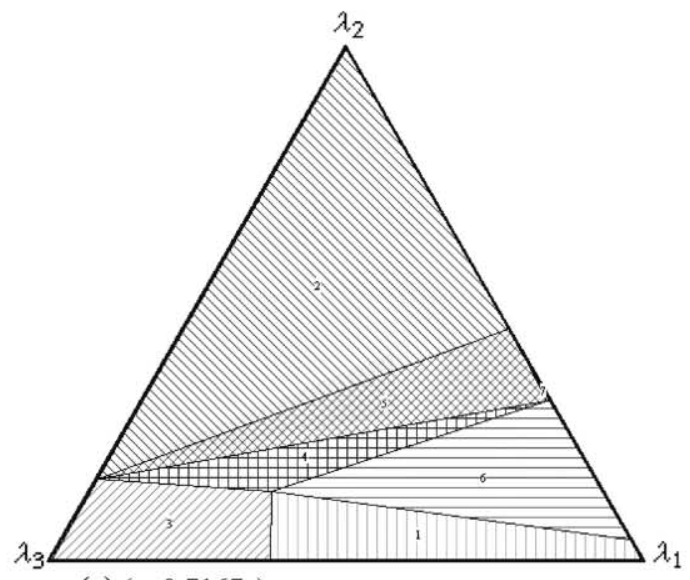

(e) $\left(y=0.7167_{\mathrm{R}}\right)$

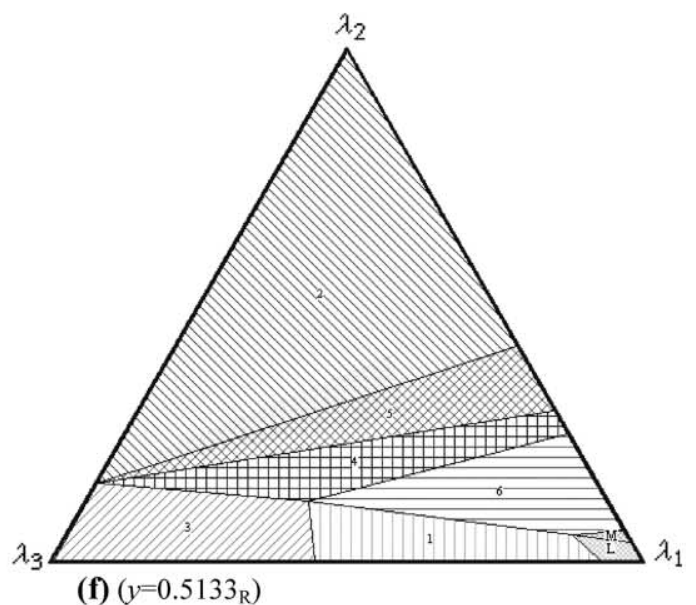

(f) $\left(y=0.5133_{\mathrm{R}}\right)$

Fig. 6. Fuzzy analysis of the objective function coefficients (efficient solutions reached with $y \geqslant 0.5133$ ): (a) $\left(y=0.5133_{\mathrm{L}}\right)$, (b) $\left(y=0.5867_{\mathrm{L}}\right),(\mathrm{c})\left(y=0.6467_{\mathrm{L}}\right),(\mathrm{d})\left(y=0.7233_{\mathrm{R}}\right),(\mathrm{e})\left(y=0.7167_{\mathrm{R}}\right),(\mathrm{f})\left(y=0.5133_{\mathrm{R}}\right)$. 


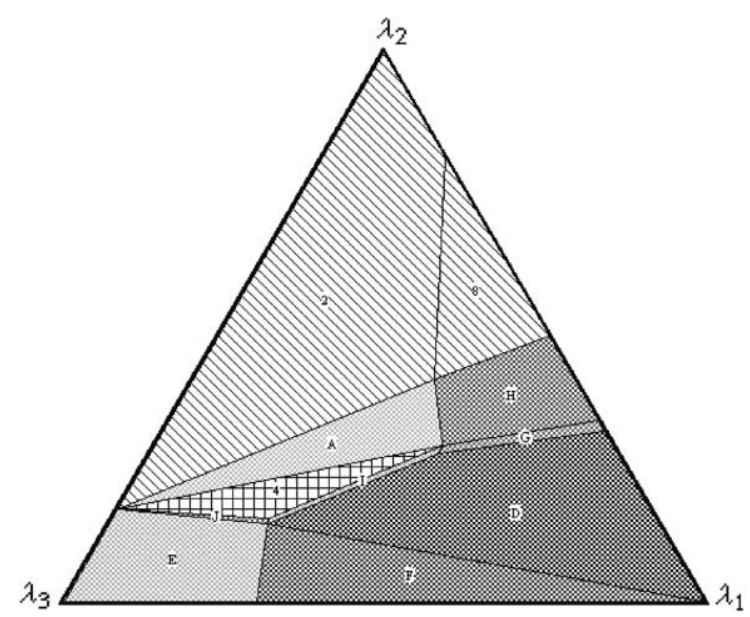

Fig. 7. Fuzzy analysis of RHS coefficients using indifference regions $\left(t=0.3367_{\mathrm{R}}\right)$.

change some of the triangular membership functions associated with these parameters (by modifying the corresponding $c_{\mathrm{L}}$ and $c_{\mathrm{R}}$ values).
An analogous study can be performed if, for example, the DM is interested in considering new fuels or other forms of electricity production, by means of the introduction of a new decision variable in the initial problem, to study how it would affect the computed basic efficient solutions.

\section{Conclusions}

Visual interactive techniques based on the analysis of indifference regions related to the weights have been developed and computationally implemented as the core of a DSS to deal with uncertainty in MOLP models by using fuzzy set theory. It must be noticed that weights are used as an operational and information exchange means and not as a way for eliciting the DM's preferences.

The DM has the possibility of interactively changing the membership functions associated with constraints and objective functions as well as

Table 4

Fuzzy analysis of the RHS coefficients $\left(t=0.3367_{\mathrm{R}}\right)$

\begin{tabular}{lrrrrrl}
\hline $\begin{array}{l}\text { Basis } \\
\left(t=0.3367_{\mathrm{R}}\right)\end{array}$ & Energy imports & $\begin{array}{l}\text { Self-production } \\
\text { of electricity }\end{array}$ & $\mathrm{CO}_{2}$ emissions & Area (\%) & Starting basis & Number of iterations \\
\hline 2 & 118603000 & 31654500 & 42445400 & 35.5831 & - & - \\
4 & 13138500 & 1668290 & 3243940 & 3.6413 & - & - \\
8 & 115908000 & 31484400 & 48484100 & 7.0706 & - & - \\
$\mathrm{A}$ & 14906300 & 2597670 & 4458920 & 6.2980 & 5 & 1 \\
$\mathrm{D}$ & 12252700 & 1274350 & 3269700 & 21.1289 & 1 or 6 & 5 or 3 \\
$\mathrm{E}$ & 12728800 & 166182 & 1280220 & 8.4277 & 3 & 5 \\
$\mathrm{~F}$ & 12252700 & 181764 & 1634470 & 9.9112 & 1 & 4 \\
$\mathrm{G}$ & 12798400 & 1598550 & 3754920 & 0.8651 & 6 & 2 \\
$\mathrm{H}$ & 14624400 & 2592540 & 5242590 & 6.3779 & 7 & 5 or 4 \\
$\mathrm{I}$ & 13132000 & 1665570 & 3244120 & 0.4887 & 1 or 6 & 6 \\
$\mathrm{~J}$ & 13137500 & 1665990 & 3240930 & 0.2028 & 3 & \\
\hline
\end{tabular}

Table 5

Fuzzy analysis of the RHS coefficients

\begin{tabular}{llll}
\hline & $t \in$ & Initial efficient basis & New efficient basis \\
\hline (i) & {$\left[0.0000_{\mathrm{L}} ; 0.9300_{\mathrm{R}}[\right.$} & $1,2,3,4,5,6,7,8$ & \\
(ii) & {$\left[0.9300_{\mathrm{R}} ; 0.9100_{\mathrm{R}}[\right.$} & $1,2,4,5,6,7,8$ & $\mathrm{E}, \mathrm{J}, \mathrm{M}$ \\
(iii) & {$\left[0.9100_{\mathrm{R}} ; 0.5333_{\mathrm{R}}[\right.$} & $2,4,5,6,7,8$ & $\mathrm{E}, \mathrm{F}, \mathrm{J}, \mathrm{L}$ \\
(iv) & {$\left[0.5333_{\mathrm{R}} ; 0.4933_{\mathrm{R}}[\right.$} & $2,4,5,6,8$ & $\mathrm{E}, \mathrm{F}, \mathrm{H}, \mathrm{J}, \mathrm{L}$ \\
(v) & {$\left[0.4933_{\mathrm{R}} ; 0.4867_{\mathrm{R}}[\right.$} & $2,4,5,8$ & $\mathrm{D}, \mathrm{E}, \mathrm{F}, \mathrm{G}, \mathrm{H}, \mathrm{I}, \mathrm{J}$ \\
(vi) & {$\left[0.4867_{\mathrm{R}} ; 0.3333_{\mathrm{R}}[\right.$} & $2,4,8$ & $\mathrm{~A}, \mathrm{D}, \mathrm{E}, \mathrm{F}, \mathrm{G}, \mathrm{H}, \mathrm{I}, \mathrm{J}$ \\
(vii) & {$\left[0.3333_{\mathrm{R}} ; 0.0000_{\mathrm{R}}\right]$} & 2,8 & $\mathrm{~A}, \mathrm{~B}, \mathrm{C}, \mathrm{D}, \mathrm{E}, \mathrm{F}, \mathrm{G}, \mathrm{H}$ \\
\hline
\end{tabular}


regarding the introduction of a new decision variable into the model. Special attention has been paid to the computational simplicity and graphical interactivity, in order to visualize dynamically the behavior of the efficient solutions according to changes in the initial model coefficients, by displaying the indifference regions. The grade of membership functions can also be interactively changed.

These techniques have been illustrated in a multiple objective input-output model, supplied with actual data for Portugal, aimed at studying the interactions between the energy sector and the economy on a national level.

\section{Acknowledgements}

This research has been partially supported by FCT under project grant 32379/99. The authors would like to thank the three anonymous referees for their helpful suggestions and constructive comments.

\section{References}

Antunes, C.H., Clímaco, J., 1992. Sensitivity analysis in MCDM using the weight space. Operations Research Letters 12, 187-196.

Antunes, C.H., Oliveira, C., Clímaco, J., 2002. A study of the interactions between the energy system and the economy using TRIMAP. In: Bouyssou, D. et al. (Eds.), Aiding Decisions with Multiple Criteria. Kluwer Academic Publishers, Dordrecht, pp. 407-427.

Borges, A.R., Antunes, C.H., 2000. Fuzzy decision aid in multiple objective linear programming. In: John, R., Birkenhead, R. (Eds.), Soft Computing Techniques and Applications. Physica-Verlag, pp. 238-243.

Carlsson, C., Korhonen, P., 1986. A parametric approach to fuzzy linear programming. Fuzzy Sets and Systems 20, 17 30 .
Chanas, S., 1983. The use of parametric programming in fuzzy linear programming. Fuzzy Sets and Systems 11, 243251.

Clímaco, J., Antunes, C.H., 1987. TRIMAP —an interactive tricriteria linear programming package. Foundations Control Engineering 12, 101-119.

Clímaco, J., Antunes, C.H., 1989. Implementation of an user friendly software package - a guided tour of TRIMAP. Mathematical and Computer Modelling 12, 1299-1309.

IPCC, 1996. Revised 1996 IPCC Guidelines for National Greenhouse Gas Inventories Reference Manual. (Downloadable from http://www.iea.org/ipcc/inv6.htm).

Leontieff, W., 1951. The Structure of the American Economy 1919-1939. Oxford University Press, New York.

Oliveira, C., Antunes, C.H., 2000. A multiobjective inputoutput model for energy planning. In: Proceedings of the 16th IMACS World Congress, Lausanne, Switzerland (in CD-ROM).

Sakawa, M., Yano, H., 1990. An interactive fuzzy satisfying method for generalized multiobjective linear programming problems with fuzzy parameters. Fuzzy Sets and Systems 35, $125-142$.

Steuer, R., 1986. Multiple Criteria Optimization: Theory, Computation and Application. Wiley, New York.

Tanaka, H., Asai, K., 1984. Fuzzy linear programming problem with fuzzy numbers. Fuzzy Sets and Systems 13, 1-10.

Werners, A.M., 1987a. An interactive fuzzy programming system. Fuzzy Sets and Systems 23, 131-147.

Werners, A.M., 1987b. Interactive multiple objective programming subject to flexible constraints. European Journal of Operations Research 31, 342-349.

Zimmermann, H.J., 1978. Fuzzy programming and linear programming with several objective functions. Fuzzy Sets and Systems 1, 45-55.

Zimmermann, H.J., 1983. Fuzzy mathematical programming. Computers Operations Research 10, 291-298.

Zimmermann, H.J., 1987. Fuzzy set, decision making, and expert systems. In: International Series in Management Science/Operations Research. Kluwer Academic Publishers, Dordrecht.

Zimmermann, H.J., 1992. Fuzzy set theory and its applications. In: International Series in Management Science/Operations Research. Kluwer Academic Publishers, Dordrecht.

Zadeh, L.A., 1965. Fuzzy Sets. Information and Control 8, 338-353.

Zeleny, M., 1982. Multiple Criteria Decision Making. McGraw-Hill, New York. 